INRA Prod. Anim., 2007, 20 (3), 213-216

\title{
Emergence des maladies chez les poissons et les mollusques : le cas des bactéries lactiques
}

\author{
D.-G. DOUET
}

Groupement de Défense Sanitaire Aquacole d'Aquitaine, 1 rue Marcel David, BP 219, F-40004 Mont-de-Marsan, France Courriel : gdsaa@wanadoo.fr

Les bactéries lactiques (qui fermentent l'acide lactique) rassemblent un large panel d'espèces. Certaines sont utilisées dans le domaine agro-alimentaire (ex : fermentation des fromages), mais d'autres sont également dotées de capacités ichthyopathogènes notables. Ces bactéries représentent un exemple significatif du phénomène d'émergence à plusieurs titres.

\section{1 / Lactococcus garvieae : un «vrai» pathogène émer- gent ?}

\section{1 / Emergence dans le temps et l'espace}

Les premiers cas de septicémie liés à Lactococcus garvieae chez les poissons ont été décrits au Japon en 1958 (Hoshina et al 1958). La maladie a ensuite été répertoriée dans un nombre croissant de pays et de continents. Les premiers cas signalés en Europe datent du tout début des années 90 en Espagne et en Italie, et de 1998 pour la France. Ces pays ont donc tous connu une augmentation de l'incidence de la maladie, pour reprendre la définition de l'émergence proposée par Toma, sans que cela soit lié à une augmentation de l'épidémiosurveillance ou à une modification des techniques de dépistage (Toma et Thiry 2003).

Il faut noter que la dénomination Lactococcus garvieae n'est que l'aboutissement des études habituelles sur les caractéristiques de la bactérie. A l'origine identifiée comme Enterococcus seriolicida (Kusuda et al 1991), elle a parfois été confondue avec Lactococcus lactis de par leur ressemblance phénotypique (Elliott et al 1991), pour finalement obtenir son identité actuelle entre 1993 et 1996 (Eldar et al 1996). Néanmoins, le chan- gement de dénomination de l'agent pathogène ne correspond pas à une émergence, puisque la maladie était installée auparavant, mais répond à la définition de «maladie nouvelle» définie par Toma et Thiry (2003).

En parallèle, si l'on remonte le temps à la recherche des premières descriptions de Lactococcus garvieae, on pourra s'étonner de la description originale de cette bactérie en dehors du milieu aquatique, dans des publications relatant des cas de mammites au Royaume-Uni (Collins et al 1983). Il semblerait donc que cette espèce bactérienne fasse preuve d'une grande «capacité d'adaptation», des vertébrés homéothermes du milieu terrestre aux vertébrés hétérothermes du milieu aquatique (on peut à ce jour l'isoler sur de multiples espèces de poissons).

Un travail a été effectué en 2004 sur le typage sérologique et biomoléculaire de souches de L. garvieae issues de pays du bassin méditerranéen (Eyngor et al 2004). Pour schématiser, les auteurs ont pu définir que l'ensemble des bactéries testées pouvait se diviser en trois groupes géographiques : un pool Italie/Israël et un pool Espagne/Grèce/Bulgarie, présentant chacun une bonne homogénéité ; et un pool rassemblant les souches françaises, souches présentant au contraire la particularité d'être éloignées des caractéristiques des deux autres pools, et par ailleurs très hétérogènes entre elles, ce qui suscite quelques commentaires :

- la dissémination géographique de la bactérie, et l'homogénéité des souches observée dans certains pays, est vraisemblablement liée, au moins pour partie, aux échanges commerciaux et à la transmission horizontale (ex : eau de transport contaminée, contact entre porteurs asymptomatiques et poissons sains) ;
- l'hétérogénéité des souches françaises semble contredire l'hypothèse de foyers reliés entre eux épidémiologiquement de façon systématique. Il semble donc que le phénomène décrit en France corresponde bien à une adaptation de la bactérie au milieu aquatique et/ou une évolution du pouvoir pathogène, phénomène qui aurait alors certainement eu lieu dans d'autres pays. Par ailleurs, les auteurs soulignent le fait que la découverte de la bactérie en France est plus récente, et que les populations ont donc été soumises moins longtemps à des facteurs de sélection environnementale et zootechnique (ex : vaccination). L'extension limitée de la maladie en foyers sporadiques, probablement liée aux mesures sanitaires prises (cf. infra), a aussi certainement influencé l'évolution des populations bactériennes.

\section{2 / Un exemple de gestion col- lective d'émergence : la lacto- coccose en France}

L'extension de la lactococcose a été foudroyante dans certains pays, qui ont été contraints d'adapter leurs pratiques zootechniques (ex : assec en été). Cette propagation inquiétante et ses conséquences économiques ont rapidement alerté les pathologistes français, qui ont effectué un grand travail de sensibilisation et de vigilance sur le terrain dès les premiers cas détectés.

Les aquaculteurs, avec l'aide des vétérinaires et des Groupements de Défense Sanitaire (GDS) Aquacoles, se sont dotés de moyens de diagnostiques performants, et ont mis en place un arsenal de mesures hygiéniques et sanitaires sur les premiers foyers bactériens (traitement, désinfection, assec, blocage des transports de poissons vivants, vaccination...), bien que la maladie ne soit pas réglementée en France. Il est intéressant de constater que la propaga- 
tion de la maladie semble s'être ralentie depuis, et que l'agent pathogène n'a pas été isolé de nouveau sur certains sites depuis des années. Le cas de la lactococcose en France est donc un bel exemple de gestion collective d'une maladie émergente par les professionnels sur le terrain. Il reste à espérer que l'évolution des conditions climatiques et du pouvoir pathogène de la bactérie, entre autres facteurs, ne favoriseront pas de nouveaux épisodes dans les années à venir, aussi convient-il de ne pas baisser la garde.

\section{2 / Emergences chez les bactéries lactiques ichthyo- pathogènes : de l'impor- tance de l'anticipation}

\section{1 / Un exemple d'outil efficace pour l'épidémiosurveillance}

L. garvieae fait partie des espèces de bactéries lactiques les plus redoutées à ce jour. Néanmoins, elle n'est pas la seule dont le pouvoir pathogène soit susceptible de se renforcer. Le quelques autres bactéries lactiques ichthyopathogènes.

Il faut également compter avec l'implication de certaines bactéries, par exemple Streptococcus iniae, dans des cas d'infections chez l'homme (Lau et al 2006).

C'est pourquoi le GDS Aquacole d'Aquitaine et le Laboratoire Départemental des Landes ont monté, en 2001, une technique biomoléculaire d'identification des principales bactéries lactiques ichthyopathogènes. Cette PCR-RFLP a été finement testée avec l'INRA de Jouy-en-Josas, et validée selon des normes AFNOR, entre 2003 et 2005, sur environ 120 souches de tableau 1 rappelle l'importance de

provenances diverses (France, Israël, Espagne, Italie...) (Pelletier et al 2005). Les professionnels disposent dorénavant d'un outil leur permettant une identification rapide, fiable, peu coûteuse, soit un véritable outil d'aide à la décision, leur permettant de mettre en place les mesures hygiéniques et sanitaires adéquates. Cette technique a également permis de vérifier l'identité des bactéries lactiques isolées sur le territoire national a posteriori, et de prouver l'absence de Streptococcus iniae parmi les isolats testés.

Un autre intérêt de cette étude a été d'apporter de nombreuses données épidémiologiques sur les bactéries rassemblées, par exemple de constater que leur spectre d'hôte n'était pas forcément aussi étroit qu'on aurait pu l'imaginer. C'est par exemple le cas de Streptococcus parauberis, isolé la plupart du temps sur le turbot, et qui a également été retrouvé sur du bar et du tilapia ; c'est également le cas de Lactococcus lactis, dont la présence récurrente parmi les isolats était inattendue.

Au final, disposer d'outils fiables tels que la PCR-RFLP permet non seulement le diagnostic de routine, mais également le suivi des maladies émergentes au quotidien.

\section{2 / Vers une émergence des zoonoses?}

La surveillance de ces bactéries lactiques, encore mal connues, reste un point important, et ce d'autant plus qu'une émergence de ces microorganismes en tant qu'agents de zoonoses est toujours à envisager. Outre 1'exemple cité auparavant concernant $S$. iniae, il faut citer la publication de Wang et al (2006), qui semble être le premier texte relatant des cas d'infections humaines à L. garvieae suite à la consommation de

Tableau 1. Exemples de bactéries lactiques ichthyopathogènes.

\begin{tabular}{|c|c|}
\hline Agent pathogène & $\begin{array}{c}\text { Preferendum thermique, } \\
\text { espèces majoritairement touchées }\end{array}$ \\
\hline Lactococcus garvieæ & $\begin{array}{c}\text { Eau chaude, nombreuses espèces } \\
\text { dont salmonidés }\end{array}$ \\
\hline Streptococcus iniæe & Eau chaude, nombreuses espèces dont tilapia \\
\hline Streptococcus parauberis & Eau chaude, turbot \\
\hline Streptococcus agalactiæ & Eau chaude, carpe, truite arc-en-ciel, tilapia \\
\hline Vagococcus salmoninarum & Eau froide, salmonidés \\
\hline Carnobacterium maltaromaticum & Eau froide, salmonidés (géniteurs) \\
\hline Lactococcus piscium & Eau froide, salmonidés \\
\hline
\end{tabular}

poissons ou fruits de mer crus et contaminés (contamination par voie orale). Les personnes infectées présentant pour la plupart des antécédents de pathologies sérieuses du tube digestif, il convient de ne pas surestimer le pouvoir zoonotique de la bactérie. Néanmoins ces informations constituent un appel à la vigilance et à la prévention, et soulignent deux aspects en particulier :

- d'une part, il est nécessaire de limiter au maximum la multiplication de ces bactéries et leur dissémination, ce qui permet de limiter leur présence dans les produits de consommation, et donc au final de limiter leur contact avec l'homme par ce biais;

- d'autre part, il semble urgent d'étudier le pouvoir pathogène de ces bactéries et de mieux connaître leur épidémiologie, afin de cerner les risques de zoonoses et leur évolution, et de prévenir une "émergence médiatique» brutale qui pourrait se révéler subjective.

\section{3 / Maîtrise des émergences : les besoins}

Il est à noter que, si peu d'informations scientifiques sont disponibles par exemple sur Vagococcus salmoninarum, les publications abordant le sujet n'en sont pas moins inquiétantes (RuizZarzuela et al 2005). Carnobacterium maltaromaticum, plutôt réputé opportuniste sur des reproducteurs post-ponte, interpelle également les vétérinaires de par ses implications éventuelles en terme de portage chronique, de lésions organiques, et de difficulté de récupération après la période de ponte. Il serait donc utile de connaître l'extension et l'évolution de ces portages (prévalence, impact sur les facteurs zootechniques...), de mener une large étude épidémiologique sur le sujet, et d'encourager les scientifiques à publier sur des descriptions de cas cliniques.

Par ailleurs, au vu de l'importance économique de la lactococcose, des travaux sur la vaccination ont rapidement démarré dans plusieurs pays. Bien que les progrès actuels soient notables, il reste encore beaucoup de travail avant de disposer de vaccins présentant une efficacité optimale et durable pour des effets secondaires minimaux, et commercialisés dans tous les pays concernés (Romalde et al 2006). Par ailleurs, l'essence d'une émergence tenant en grande partie à son imprévisibilité, il sera a priori toujours indispensable de disposer d'outils de lutte tels que les antibiotiques, que ce soit contre de nouveaux agents pathogènes lac- 
tiques, contre des variants n'autorisant pas de protection croisée avec les vaccins déjà existants, ou en cas de rupture d'immunité. Les bactéries lactiques ayant une fâcheuse tendance à la multirésistance, et l'arsenal thérapeutique disponible en France étant très maigre, il est urgent d'envisager un travail sur la disponibilité immédiate de matières actives, efficaces et en quantité suffisante, que les vétérinaires pourront éventuellement prescrire selon le principe de la cascade. L'objectif est de favoriser la lutte dans les pays où la maladie est encore présente, et d'avoir des moyens de réactions immédiats en cas d'émergence ou de rééemergence dans les autres.

De façon plus globale, il semble important de mettre en commun les connaissances et outils dont disposent les différents pays confrontés à ces maladies, ce que le GDS Aquacole d'Aquitaine envisage de mettre en place un colloque sur le sujet en septembre 2007 à Biarritz (http://aquafilia.fr/biarritz2007).

\section{Conclusion}

Les bactéries lactiques restent un sujet de préoccupation majeure à ce jour. Malgré le développement de travaux de recherche assidus dans plusieurs pays, et la mobilisation des professionnels, la gestion sur le terrain reste ardue et doit en permanence s'adapter au contexte épidémiologique. Après l'émergence et la propagation rapide de la lactococcose en particulier, et malgré l'efficacité de mesures mises en place en France par exemple, il est difficile d'augurer de l'évolution de ce type de pathologies, tant du point de vue des élevages piscicoles que de la santé humaine. La vigilance et l'anticipation restent donc de mise.

\title{
Références
}

Collins M.D., Farrow J.A., Phillips B.A., Kandler O., 1983. Streptococcus garvieae sp. nov. and Streptococcus plantarum sp. nov. J. Gen. Microbiol., 129 (11), 3427-3431.

Eldar A., Ghittino C., Asanta L., Bozzetta E., Goria M., Prearo M., 1996. Enterococcus seriolicida is a junior synonym of Lactococcus garvieae a causative agent of septicemia and meningoencephalitis in fish. Curr. Microbiol., 32, 85-88.

Elliott J.A., Collins M.D., Pigott N.E., Facklam R.R., 1991. Differentiation of Lactococcus lactis and Lactococcus garvieae from humans by comparison of whole-cell protein patterns. J. Clin. Microbiol., 29 (12), 2731-2734.

Eyngor M., Zlotkin A., Ghittino C., Prearo M., Douet D.G., Chilmonczyk S., Eldar A., 2004. Clonality and diversity of the fish pathogen Lactococcus garvieae in mediterranean countries. Appl. Environ. Microbiol., 70, 5132-5137.
Hoshina T., Sano T., Morimoto Y.A., 1958. A streptococcus pathogenic to fish. J. Tokyo Univ. Fish, 44, 57.

Kusuda R., Kawai K., Salati F., Banner C.R., Fryer J.L., 1991. Enterococcus seriolicida sp. nov., a fish pathogen. Int. J. Syst. Bacteriol., 41, 406-409.

Lau S.K., Woo P.C., Luk W.K., Fung A.M., Hui W.T., Fong A.H., Chow C.W., Wong S.S., Yuen K.Y., 2006. Clinical isolates of Streptococcus iniae from Asia are more mucoid and beta-hemolytic than those from North America. Diagn. Microbiol. Infect. Dis., Mar, 54, 3, 177-181.

Pelletier C., Michel C., Tailliez P., Bellet C., Beyrie M., Lautraite A., Douet D.G., 2005. Improvement of PCR-RFLP identification of lactic acid bacteria isolated from healthy and diseases fish. Coll. Int. New Diagnostic Technology, 3-5 Octobre 2005, Saint-Malo, France, Poster.
Romalde J.L., Ravelo C., Toranzo A.E., 2006. Control of fish lactococcosis : efficacy of vaccination procedures. CAB Reviews: Perspectives in agriculture, Veterinary Science, Nutrition and Natural Resources, January 2006, 1, n006, 1-6.

Ruiz-Zarzuela I., de Bias I., Gironés O., Ghittino C., Múzquiz J.L., 2005. Isolation of Vagococcus salmoninarum in Rainbow Trout, Oncorhynchus mykiss (Walbaum), Broodstocks: characterization of the pathogen. Vet. Res. Com., 29, 553-562.

Toma B., Thiry E., 2003. Qu'est-ce qu'une maladie émergente ? Epidemiol. Santé Anim., 44, 1-11.

Wang C.Y.C., Shie H.S., Chen S.C., Huang J.P., Hsieh I.C., Wen M.S., Lin F.C. Wu D., 2006. Lactococcus garvieae infections in humans: possible association with aquaculture outbreaks. Int. J. Clin. Pract., 61, 68-73.

\section{Résumé}

Certaines bactéries lactiques sont des agents pathogènes redoutés de la filière piscicole. La lactococcose, en particulier, constitue une bonne illustration de maladie émergente, dont la gestion a été plus ou moins heureuse selon les pays. Si certains outils diagnostiques performants sont désormais disponibles, il convient toutefois de se préoccuper davantage des mesures de traitement et de prophylaxie, et de mobiliser les compétences internationales sur le sujet.

\begin{abstract}
Emerging diseases in fishes and molluscs: the case of lactic bacteria

In the field of fish farming, some lactic bacteria are feared pathogens. Lactococcosis especially is a perfect illustration of an emerging disease whose management is more or less successful according to countries. Nowadays, even if some efficient diagnostic tools are available, one should pay special attention to treatment and prophylaxis measures. In addition, international skills on this subject must be mo-
\end{abstract} bilised.

DOUET D.-G., 2007. Emergence des maladies chez les poissons et les mollusques : le cas des bactéries lactiques. INRA Prod. Anim., 20, 213-216. 
